\title{
The spectrum of hemophagocytic lymphohistiocytosis: a retrospective study comparing adult macrophage activation syndrome to malignancy-associated hemophagocytic lymphohistiocytosis
}

\author{
S. D. Good ${ }^{1}$ (D) . S. D. Wade ${ }^{2}$ (D) . V. C. Kyttaris ${ }^{2}$ (D)
}

Received: 11 October 2021 / Accepted: 30 December 2021 / Published online: 11 January 2022

(C) The Author(s), under exclusive licence to Springer-Verlag GmbH Germany, part of Springer Nature 2022

\begin{abstract}
Secondary hemophagocytic lymphohistiocytosis (sHLH) is a life-threatening inflammatory syndrome that can be triggered by autoimmune diseases, malignancy, or infection. In rheumatologic patients, sHLH is referred to as macrophage activation syndrome (MAS). Differentiating between triggers is important for prompt treatment and prognosis. Data comparing subsets of sHLH are limited due to the rarity of this disease. We aim to explore differences in clinical features that may differentiate MAS from malignancy-associated HLH (mHLH) patients. We conducted a single-center retrospective study assessing clinical characteristics, laboratory parameters, treatment regimens and outcomes in 34 patients with sHLH over a 16 year period. We compared patients with MAS to those with mHLH. Hepatomegaly was not present in the MAS group but was present in the mHLH group ( 0 vs. $25 \%, p=0.024)$. MAS patients had on average nearly double the concentration of platelets at 50.0 (IQR: $31.0-78.0 \mathrm{K \mu} / \mathrm{L}$ ) vs. $29.0 \mathrm{~K} \mu / \mathrm{L}$ (IQR: $14.0-37.5 \mathrm{~K} \mu / \mathrm{L}$ ), $p=0.003$. Soluble IL-2R concentrations were four times lower in the MAS group with a median soluble IL-2R concentration of $6814.5 \mathrm{kU} / \mathrm{L}$ (IQR: 2101-2610 kU/L) vs. $27972.0 \mathrm{kU} / \mathrm{L}$ (IQR: $12,820-151,650 \mathrm{kU} / \mathrm{L}$ ), $p=0.010$. The MAS group fared better overall than the mHLH group but was not statistically significant (mortality 22 vs. $44 \%, p=0.18$ ). MAS and mHLH patients exhibited different laboratory parameters and clinical features, most notably differences in platelet counts, soluble IL-2R concentration and hepatomegaly, which may help differentiate these conditions early in their course.
\end{abstract}

Keywords Macrophage activation syndrome · Lymphohistiocytosis, hemophagocytic $\cdot$ Lupus erythematosus, systemic · Still's disease, adult-onset

\section{Introduction}

Secondary hemophagocytic lymphohistiocytosis (sHLH) is a syndrome characterized by disordered immune response to a variety of triggers, including infection or malignancy, that results in profound inflammation and can be potentially life threatening. Features most frequently associated with sHLH include fever, lymphadenopathy, hepatosplenomegaly, pancytopenia, elevated ferritin, and elevated soluble IL-2

S. D. Good

Sgood@bidmc.harvard.edu

1 Department of Medicine, Beth Israel Deaconess Medical Center, Harvard Medical School, 330 Brookline Ave, Boston, MA 02215, USA

2 Division of Rheumatology and Clinical Immunology, Beth Israel Deaconess Medical Center, Harvard Medical School, Boston, MA, USA receptor. When this disease process occurs in the setting of rheumatologic diseases, it is called macrophage activation syndrome (MAS). MAS is most commonly associated with adult-onset Still's disease (AOSD), but can also rarely be seen in other rheumatologic conditions, including systemic lupus erythematosus (SLE) and dermatomyositis [1]. Malignancies known to trigger sHLH include lymphomas, T/NK-cell disorders, acute leukemias, lymphoproliferative diseases, and myelodysplastic syndrome [2]. Although both malignancy-associated HLH (mHLH) and MAS have poor outcomes, MAS carries a lower fatality rate $[1,2]$.

Primary HLH was first described as a genetic, inherited condition. The exact mechanism that triggers MAS is unknown, but it is likely related to a defect in immune cell cytolytic activity, which results in prolonged cell-to-cell interaction and a pro-inflammatory cytokine cascade [3, 4]. In recent years, certain loss of function mutations were retroactively identified in patients who developed sHLH as 
an adult, showing that there is indeed a genetic component to these acquired syndromes [5]. In addition to an underlying genetic predisposition, sHLH requires an environmental trigger, such as infection, immunosuppression, uncontrolled autoimmune disease, or malignancy [6]. Identifying the trigger is important, because treatment differs depending on the underlying etiology and patients often present in critical condition $[7,8]$.

There are several validated diagnostic tools used to aid in the diagnosis of MAS and HLH. The $\mathrm{H}$ score is one such commonly used example [9]. The cohort used to develop the $\mathrm{H}$ score, however, consisted of a paucity of rheumatologic cases. Similarly, the HLH-2004 set of criteria, which is the most frequently cited in MAS/HLH literature [10], was largely based on a pediatric population of patients with familial HLH [11].

In one significant prior attempt to differentiate MAS from other similar clinical syndromes, Daví et al. delivered surveys to clinical experts in pediatric rheumatology to identify commonly recognized features of MAS [12]. Respondents most frequently associated macrophage hemophagocytosis on bone marrow biopsy, hyperferritinemia, persistent fever, thrombocytopenia, and increased soluble IL-2 receptor, amongst others, with MAS. In other studies, an elevated ferritin-to-ESR ratio has been proposed as a tool for diagnosing MAS [13].

MAS is often difficult to distinguish from other significant disease states, including sepsis, disseminated intravascular coagulation (DIC) and, particularly in SLE, a flare of underlying rheumatologic disease. Furthermore, within MAS, there exist unique clinical features to MAS secondary to SLE when compared to MAS secondary to AOSD [14-16]. As a result, MAS diagnosis may be delayed or missed altogether, leading to poor outcomes [17]. Further research on features unique to MAS and mHLH is essential for early diagnosis and proper treatment. In this manuscript, we identify clinical features that may help distinguish autoimmune-associated MAS patients in adults from malignancy-associated HLH cases.

\section{Methods}

\section{Study design}

We performed a retrospective study looking at outcomes in patients with sHLH at an academic tertiary care hospital in Boston, MA. We identified patients using ICD9/10 codes for HLH/MAS from 2004 to 2020 (ICD9 288.4, ICD 10 D76.1), and/or patients with high ferritin levels and fever who were cross referenced with SLE, AOSD, inflammatory myopathy, inflammatory arthritis or hematologic malignancy. The study was conducted with the approval of an institutional review board and followed the Strengthening the Reporting of Observational Studies in Epidemiology (STROBE) reporting guideline.

\section{Inclusion and exclusion criteria}

From the patients identified, we included those who fulfilled diagnostic criteria for a rheumatologic condition or hematologic malignancy and HLH. A diagnosis of MAS/HLH required fulfillment of the HLH-2004 classification criteria or a clinical diagnosis by an expert consultant in combination with a high probability H-score. We excluded MAS/ HLH cases triggered by sepsis. Children were excluded from this study as the focus was adult-onset MAS, which is not as well characterized as its pediatric form that is typically associated with juvenile inflammatory arthritis.

Per the HLH-2004 diagnostic protocol, patients in this study needed to meet 5 of the following criteria: fever, splenomegaly, bicytopenia, hypertriglyceridemia and/or hypofibrinogenmia, hemophagocytosis, low/absent NK-cell-activity, hyperferritenmia and high soluble IL-2 receptor levels [11]. We broadened our search to include patients with high probability $\mathrm{H}$ scores plus diagnosis by an expert documented in the chart to allow for inclusion of patients who did not meet HLH-2004 criteria as a result of missing data at their initial work-up. H-score criteria are similar to HLH-2004 criteria but also include underlying immunosuppression and liver function. We searched for patients with laboratory features seen in MAS or HLH with known underlying rheumatologic conditions or hematologic malignancy to find patients who may not have been properly coded for MAS or HLH. A diagnosis of SLE or AOSD required confirmation from an attending physician documented in the chart based on American College of Rheumatology (ACR) diagnostic criteria [18] and Yamaguchi criteria [19], respectively. Of the dermatomyositis patients, one was diagnosed by muscle biopsy, while the other was diagnosed by typical exam findings (heliotrope rash, Gottron papules) along with elevated creatine kinase (CK), consistent with 2017 EULAR/ACR diagnostic criteria [20]. All malignancies were diagnosed by a hematology/oncology attending physician.

\section{Clinical and laboratory data}

We reviewed each chart once patients were deemed to meet the above criteria, and extracted information on patient demographics, medical history, clinical presentation, treatment and outcomes. We also collected SLE and AOSD patient baseline disease characteristics, including systemic lupus erythematosus disease activity index (SLEDAI) criteria [21], Yamaguchi criteria, select immunologic laboratory data, vital signs, clinical features and radiographic reports. 


\section{Statistical analysis}

We compared MAS cases to mHLH controls using STATA. We utilized Wilcoxon rank sum tests for categorical analysis, because our data were not normally distributed. As such, all $p$ values are derived from this non-parametric analysis tool and 25th to 75th interquartile ranges are reported. A $p$ value less than 0.05 was regarded as statistically significant.

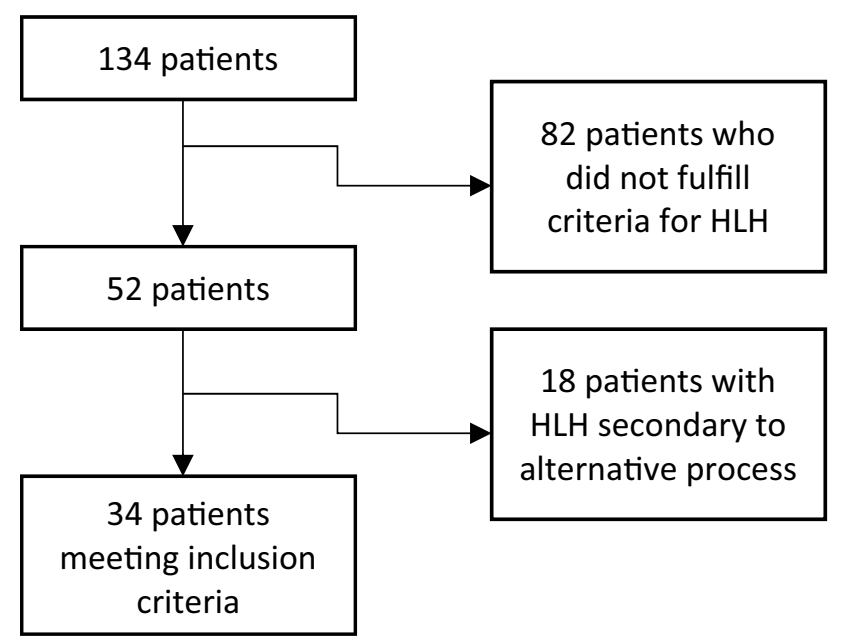

Fig. 1 Patients meeting inclusion criteria for the study. Figure made using Microsoft Word
Missing data for key laboratory values were coded as missing and were not imputed.

\section{Results}

\section{Patient demographics, clinical data}

Of the 134 charts that we reviewed, 34 met inclusion criteria for the study (see Fig. 1). Demographics and clinical data, including laboratory values and baseline patient characteristics are described in Table 1.

Overall, 8 of 18 MAS patients had SLE as their underlying diagnosis, and 6 had AOSD as their underlying diagnosis. Of the remaining four patients, two had dermatomyositis, one had rheumatoid lung disease, and one had rheumatoid arthritis as underlying conditions for MAS.

For SLE patients, we collected ACR criteria data from patient charts at time of initial presentation of SLE. Cutaneous manifestations were present in 7 (87.5\%) patients, leukopenia present in $6(75 \%)$, thrombocytopenia present in 5 $(62.5 \%)$, arthritis present in $4(50 \%)$, serositis present in 2 (25\%), and renal disease present in $2(25 \%)$ at time of SLE diagnosis. A total of seven out of eight patients $(87.5 \%)$ had a history of hypocomplementemia. All SLE patients were ANA positive. Extractable nuclear antigen antibody profiles revealed five $(62.5 \%)$ patients with anti-RNP antibodies,

Table 1 Patient characteristics at time of admission (median, 25th and 75th percentile ranges in brackets) and pre-treatment immunologic lab values on presentation in MAS vs. mHLH patients

\begin{tabular}{|c|c|c|c|}
\hline & MAS $(n=18)$ & $\mathrm{mHLH}(n=16)$ & $p$ value \\
\hline \multicolumn{4}{|l|}{ Patient demographics } \\
\hline Sex (female) & $14(77 \%)$ & $7(44 \%)$ & 0.042 \\
\hline Age at diagnosis of autoimmune disease or malignancy (years) & $34.5[28,46]$ & $65.5[44,77]$ & $<0.001$ \\
\hline Age at MAS/mHLH diagnosis (years) & $35.5[30,47]$ & $70.0[46.5,77]$ & 0.002 \\
\hline Time between autoimmune/malignancy diagnosis and HLH (months) & $2.5[0,48]$ & $0.0[0,14]$ & 0.22 \\
\hline HLH as initial presentation of underlying disease & $9(50 \%)$ & $11(69 \%)$ & 0.27 \\
\hline \multicolumn{4}{|l|}{ Clinical Features } \\
\hline Hepatomegaly & $0(0 \%)$ & $4(25 \%)$ & 0.024 \\
\hline Splenomegaly & $4(22 \%)$ & $8(50 \%)$ & 0.091 \\
\hline Fevers & $17(94 \%)$ & $13(81 \%)$ & 0.23 \\
\hline \multicolumn{4}{|l|}{ Baseline (pre-treatment) immunologic lab values } \\
\hline WBC $(\mathrm{K} \mu / \mathrm{L})$ & $3.9[1.2,5.8]$ & $1.6[0.4,3.8]$ & 0.026 \\
\hline $\operatorname{ANC}(\mathrm{K} \mu / \mathrm{L})$ & $4[0.7,5.5]$ & $0.3[0,2.1]$ & 0.005 \\
\hline $\mathrm{Hgb}(\mathrm{g} / \mathrm{dL})$ & $8.4[7.8,10.4]$ & $7.2[6.7,7.8]$ & 0.008 \\
\hline Platelets $(K \mu / L)$ & $50[31,78]$ & $29.9[14,37.5]$ & 0.003 \\
\hline Fibrinogen (mg/dL) & $147.5[127,234]$ & $123[94,276]$ & 0.33 \\
\hline Triglycerides (mg/dL) & $276[147,345]$ & $351[243,578.5]$ & 0.13 \\
\hline Ferritin $(\mathrm{ng} / \mathrm{mL})$ & $12,016[3032,32068]$ & $14,578[10486,24083]$ & 0.28 \\
\hline AST (U/L) & $580[239,1317]$ & $244.5[90,852.5]$ & 0.17 \\
\hline Soluble IL-2R (kU/L) & $6814.5[2101,7610]$ & $27,972.0[12820,151650]$ & 0.01 \\
\hline
\end{tabular}


three $(37.5 \%)$ patients with anti-Smith antibodies, two (25\%) patients with positive anti-phospholipid antibodies (defined as positive lupus anticoagulant or high titer anticardiolipin or anti-beta-2-glycoprotein-1 antibodies), and 2 (25\%) patients with anti-Ro.

We collected Yamaguchi criteria data for AOSD patients. During their disease course, all AOSD patients (6 of 6) presented with high fevers, arthritis/arthralgias, characteristics rash, and leukocytosis. We noted that four (67\%) patients presented with sore throat and LFT abnormalities, three (50\%) presented with lymphadenopathy, $2(33 \%)$ presented with splenomegaly. Of the AOSD patients, five of six (83\%) had negative RF, and three of six (50\%) had negative ANA, one did not have ANA checked, and two had weakly positive ANA with 1:40 and 1:80 titers that were considered to be clinically insignificant.

In Table 1, we summarize baseline clinical characteristics and immunologic lab value data from time of admission. Hepatomegaly was not present in MAS compared to the mHLH group ( 0 vs. $25 \%, p=0.024)$. MAS patients had less severe cytopenias compared to mHLH patients: with median $\mathrm{WBC}$ of $3.9 \mathrm{~K} \mu / \mathrm{L}$ (IQR: $1.2,5.8 \mathrm{~K} \mu / \mathrm{L}$ ) vs. $1.6 \mathrm{~K} \mu / \mathrm{L}$ (IQR: $0.4,3.8 \mathrm{~K} \mu / \mathrm{L}$ ), $p=0.026$; median ANC $4.0 \mathrm{K \mu} / \mathrm{L}$ (IQR: $0.7-5.5 \mathrm{~K} \mu / \mathrm{L}$ ) vs. $0.3 \mathrm{K \mu} / \mathrm{L}$ (IQR: $0.0-2.1 \mathrm{~K} \mu / \mathrm{L}), p=0.005$; median hemoglobin $8.4 \mathrm{~g} / \mathrm{dL}$ (IQR 7.8-10.4 g/dL) vs. $7.2 \mathrm{~g} / \mathrm{dL}$ (IQR: $6.7-7.8 \mathrm{~g} / \mathrm{dL}$ ), $p=0.008$; and median platelets $50.0 \mathrm{~K} \mu / \mathrm{L}$ (IQR: 31.0-78.0 $\mathrm{K} \mu / \mathrm{L}$ ) vs. $29.0 \mathrm{~K} \mu / \mathrm{L}$ (IQR: $14.0-37.5 \mathrm{~K} \mu / \mathrm{L}), p=0.003$. Soluble IL-2R concentrations were on average 4 times lower in the autoimmune vs. the malignancy group: median $6814.5 \mathrm{kU} / \mathrm{L}$ (IQR: $2101-2610 \mathrm{kU} / \mathrm{L}$ ) vs. 27972.0 kU/L (IQR: 12,820-151,650 kU/L), $p=0.010$. All other admission clinical and laboratory characteristics between groups did not reach statistical significance. Of note, dermatomyositis patients carry a higher risk than the general population for certain malignancies, but no dermatomyositis patient in this group was diagnosed with malignancy during the study period.

\section{Hospital course and outcomes}

Data for patient hospital course and outcomes are summarized in Table 2. MAS patients spent less than a third of the amount of days in the hospital (12.5 [10-22] vs. 38.5 [22-47], $p<0.001$ ), required less transfusions ( $10 \mathrm{vs}$ $16, p=0.001)$, and had better recovery of platelets after treatment (212.0 [54-235] vs. 52.0 [18-120.5], $p=0.034)$ when compared to HLH patients (Fig. 2). The mortality rate was high in both groups with the MAS patients faring overall better than mHLH patients but that difference did not reach statistical significance ( 22 vs. $44 \%, p=0.18$ ).

Of the four patients who died in the MAS group, one had AOSD, one had SLE, one had dermatomyositis, and one had rheumatoid lung disease as their underlying diagnoses. The causes of death included shock $(n=2)$, renal failure $(n=1)$ and encephalopathy $(n=1)$. In the mHLH group, there were seven deaths with the causes including sepsis $(n=2)$, shock $(n=3)$, encephalopathy $(n=1)$ and renal failure $(n=1)$. Autopsy was performed in only one patient from the mHLH group. The immediate cause of death was initially identified as sepsis; however, on pathology review this was reclassified as secondary HLH in the setting of florid tissue hemophagocytosis, profound anemia, thrombocytopenia, elevated trigycerides and hyperferritinemia.

Treatment regimens also differed between the groups. Pulse dose steroids (defined as methylprednisolone $1000 \mathrm{mg}$ daily for at least 3 days) were utilized more frequently in MAS patients compared to HLH patients ( 78 vs. $12 \%$, $p<0.001)$. Conversely cyclophosphamide was utilized less in the MAS group (11 vs. 50\%, $p=0.013$ ), as was etoposide/dexamethasone combination treatments ( 0 vs. $44 \%$, $p=0.002$ ).

\section{Concomitant infections}

Patients who were thought to have sepsis as the underlying etiology of their HLH presentation were excluded from this study, which specifically assessed individuals with rheumatologic or malignant etiologies of disease. However, several patients included in this study developed sepsis during their hospital course. Of those patients, 11 of 34 were treated for a bacterial infection, including pneumonia, urinary tract infection, cellulitis and bacteremia. EBV PCR was assessed in 21 of the 38 patients during their hospital admission and found to be positive in $10(47.6 \%)$ of these patients. Treatment for EBV was only offered in 3 of those 10 patients, all of which were from the mHLH group. Two patients were given rituximab and one bortezomib for EBV. CMV PCR was elevated in three patients overall (one from MAS and two from mHLH), but only one patient (from the MAS group) received treatment for CMV during their hospital course. CMV viremia was not consistently measured. CMV IgM was negative in all 21 of the patients in which it was assessed.

\section{Discussion}

In this cohort, we found that patients with MAS tended to be younger, required less transfusions and had shorter hospital stays than mHLH patients. Despite these favorable differences, $22 \%$ of patients with MAS in our study died. Even higher numbers of mHLH patients (44\%) died during their hospitalization. Thus, early recognition and appropriate treatment could be critical to improve mortality. Our findings compared to other published series show somewhat higher 
mortality rates in MAS and overall better than reported survival in $\mathrm{mHLH}$. Though reported mortality rates vary greatly between studies, HLH associated with lymphomas has been reported as having a significantly lower overall survival [22].

A total of 20 out of the 34 patients did not have a previously established autoimmune or malignant disease, but rather developed HLH during the initial presentation of their systemic disease. MAS was inaugural in 50\% of the autoimmune disease population studied here, which is slightly higher than previous data on timing of MAS in disease course [14]. Interestingly, there were more cases of SLEassociated MAS than Still's-associated MAS in our cohort despite this being an underrecognized complication of SLE. Although there are limited studies assessing the features of SLE-associated MAS in the adult population $[14,15]$, in pediatric populations, it has been reported as high as $9 \%$ of SLE patients developed MAS with fever, high ferritin and more pronounced cytopenias as important differentiating factors from SLE flare [23].

Organomegaly was less common in MAS patients, compared to mHLH patients in our cohort. It has to be noted that organomegaly and cytopenia are hallmark features of many of the malignancies seen in mHLH patients in this study. In rheumatic patients, hepatomegaly is part of the diagnostic criteria for AOSD but not part of SLICC or ACR/EULAR diagnostic criteria for SLE. It is unclear what percentage of hepatomegaly is truly due to $\mathrm{MHLH}$ presentation as opposed to underlying malignancy, but multiple pediatric and adult population studies of HLH have shown hepatomegaly to be a key component of the disease [24, 25].

Thrombocytopenia, anemia and elevations in soluble IL-2R were less pronounced in MAS patients compared to the HLH patients, which is consistent with prior studies [26]. Previously, IL-2R, ferritin and the IL-2R-to-ferritin

Table 2 Hospital course, post-treatment immunologic lab values, treatment and outcomes for MAS and mHLH patients (median, 25th and 75th percentile ranges in brackets)

\begin{tabular}{|c|c|c|c|}
\hline & MAS $(n=18)$ & $\mathrm{mHLH}(n=16)$ & $p$ value \\
\hline \multicolumn{4}{|l|}{ Hospital course } \\
\hline Pressor requirement & $8(47 \%)$ & $5(31 \%)$ & 0.35 \\
\hline $\begin{array}{l}\text { Hypoxia requiring supplemen- } \\
\text { tal oxygen }\end{array}$ & $9(53 \%)$ & $11(69 \%)$ & 0.35 \\
\hline Development of ARDS & $2(12 \%)$ & $2(12 \%)$ & 0.95 \\
\hline Days in hospital & $12.5[10,22]$ & $38.5[27,47]$ & $<0.001$ \\
\hline Days in ICU & $2.5[0,11]$ & $2[0,9]$ & 0.97 \\
\hline Required intubation & $7(39 \%)$ & $5(31 \%)$ & 0.64 \\
\hline Required transfusion & $10(56 \%)$ & $16(100 \%)$ & 0.002 \\
\hline \multicolumn{4}{|c|}{ Post-treatment immunologic lab values } \\
\hline WBC $(\mathrm{K} \mu / \mathrm{L})$ & $5.2[3.3,13.4]$ & $4.9[1.6,18.8]$ & 0.69 \\
\hline Platelets $(K \mu / L)$ & $212[54,235]$ & $52[18,120.5]$ & 0.034 \\
\hline Fibrinogen (mg/dL) & $198[133,324]$ & $229[158,296]$ & 0.65 \\
\hline ALT (U/L) & $128[55,266]$ & $62[38,179]$ & 0.17 \\
\hline \multicolumn{4}{|l|}{ Treatment } \\
\hline Pulse steroids & $14(78 \%)$ & $2(12 \%)$ & $<0.001$ \\
\hline $\begin{array}{l}\text { Cumulative steroid dose over } \\
\text { hospitalization (mg equiva- } \\
\text { lents of methylprednisolone) }\end{array}$ & $3470[3000,4003]$ & $1432.7[913.6,2407.6]$ & 0.016 \\
\hline Cyclophosphamide & $2(11 \%)$ & $8(50 \%)$ & 0.013 \\
\hline MMF & $1(6 \%)$ & $0(0 \%)$ & 0.34 \\
\hline Etoposide/dexamethasone & $0(0 \%)$ & $7(44 \%)$ & 0.002 \\
\hline IVIG & $4(22 \%)$ & $0(0 \%)$ & 0.045 \\
\hline Anakinra & $6(33 \%)$ & $0(0 \%)$ & 0.011 \\
\hline \multicolumn{4}{|l|}{ Outcomes } \\
\hline Discharged & $14(78 \%)$ & $9(56 \%)$ & 0.18 \\
\hline Death & $4(22 \%)$ & $7(44 \%)$ & 0.18 \\
\hline $\begin{array}{l}\text { SLE/AOSD quiescent at } \\
1 \text { year follow-up? }\end{array}$ & $5(42 \%)$ & - & \\
\hline $\begin{array}{l}\text { Repeat episode of MAS at any } \\
\text { point? }\end{array}$ & $0(0 \%)$ & $2(25 \%)$ & 0.043 \\
\hline
\end{tabular}



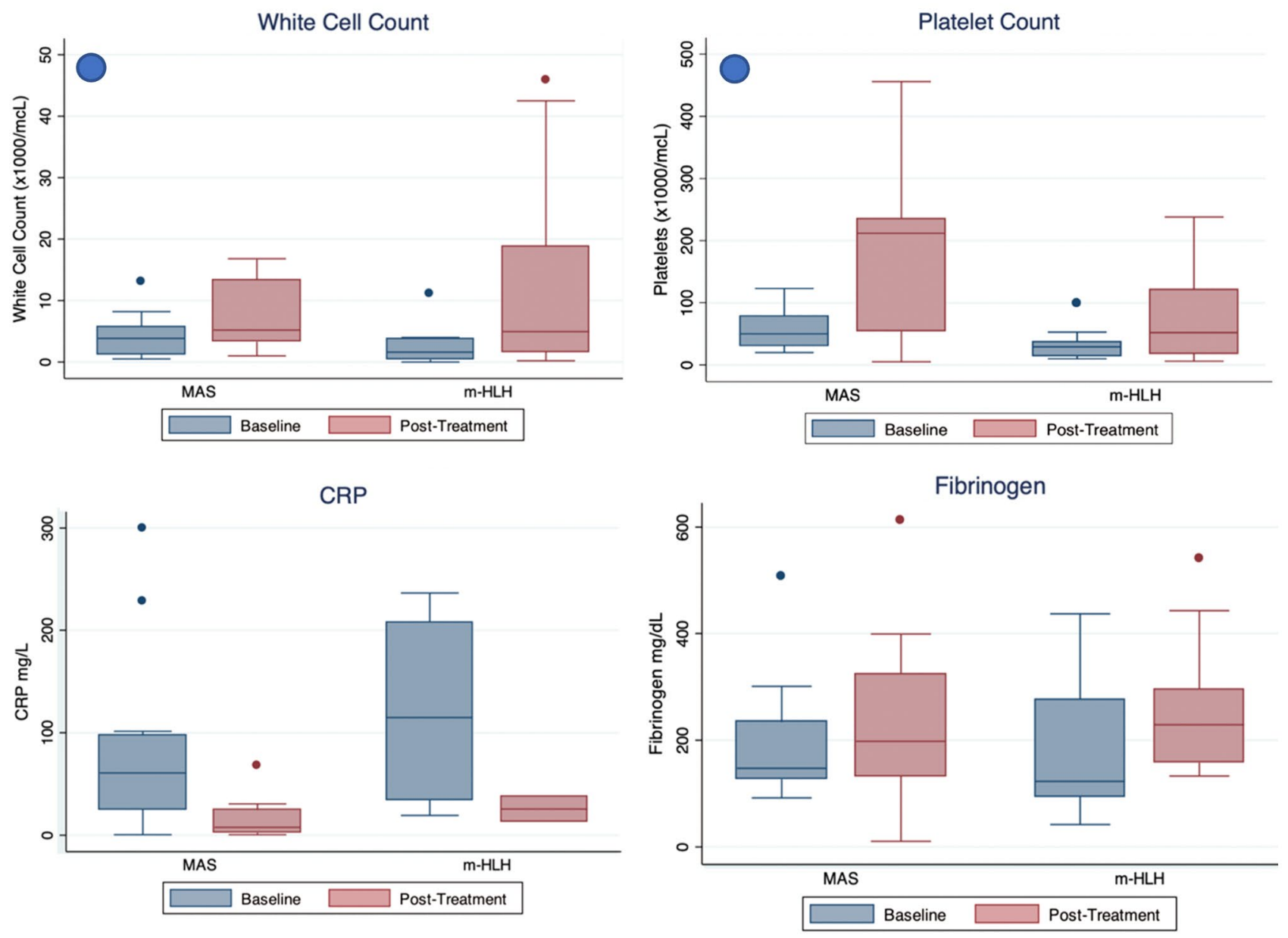

Fig. 2 Pre-treatment and post-treatment select immunologic measurements in comparison. Clockwise from top left, changes in WBC count, platelet count, fibrinogen and CRP before and after treatment.

ratio had been proposed as important markers for diagnosis of MAS and mHLH [27, 28]. Interestingly, in our study, ferritin did not differ between the two groups despite inclusion of AOSD patients where high ferritin is common. In previous studies, it has also been suggested that better prognosis is associated with a rapid drop in ferritin levels [29]; in our study since pre- and post-treatment ferritin was not consistently assessed, we could not replicate this finding. IL-2R concentrations did differ between groups, significantly. Furthermore, not only were platelet counts significantly higher in the MAS cohort at $50 \mathrm{~K} \mu / \mathrm{L}$ compared to $29.9 \mathrm{~K} \mu / \mathrm{L}$, but they also showed a significant difference in post-treatment values between the two groups. Platelet discrepancies between MAS and other forms of HLH have been reported in the past, and show MAS patients as having higher platelet counts compared to other groups [26]. These studies, however, predominantly compare MAS to familial forms of HLH. Since there is little data on predicting unique

Bars represent interquartile ranges for data analyzed using Wilcoxon rank sum tests. Circle in upper left corner indicates statistical significance between MAS and mHLH groups. Figure made using STATA

characteristics to the adult population of MAS, our findings may be helpful in differentiating MAS from mHLH.

With respect to treatment regimens, MAS patients were more likely to receive pulse dose steroids and immunomodulatory medications compared to mHLH patients, while mHLH patients were more likely to receive etoposide/dexamethasone combination therapy. Etoposide/ dexamethasone-based therapies are reported as resulting in a long-term survival of greater than $50 \%$ in familial forms of HLH [30]. Though there are limited randomized control trials for MAS treatment protocols, the treatments seen in this study are similar to what is seen in the literature [30-32]. Biologics are increasingly being used in patients with severe MAS [33], and in our cohort, Anakinra was used in 33\% of MAS cases. Though there is minimal data on the use of immunologic therapies in MAS in an adult population, early pediatric studies on the use of anakinra in corticosteroid refractory MAS have been promising [34]. There is limited data to support the 
use of immunomodulatory medications as monotherapy, including IL-6 [35].

It is frequently unclear what tips somebody into the immune dysregulation that causes MAS or mHLH, but infection can be a trigger for these patients. Viral triggers, including EBV, CMV, parvovirus, HSV, HIV and, more recently, SARS-CoV-2, have been implicated in the development of secondary HLH in the absence of an underlying malignancy or autoimmune disease [36]. Bacterial infections, including anaplasma and ehrlichia, are also associated with HLH $[37,38]$. EBV and CMV PCR was not consistently evaluated in our cohort; however, a quarter of patients had detectable EBV by PCR and three patients were found to have CMV viremia. EBV is a common trigger of $\mathrm{HLH}$, including in patients with no underlying autoimmune disease or malignancy, and, similar to the patients in this cohort, EBV-associated HLH is frequently treated with rituximab in addition to etoposide/dexamethasone-based treatments [39]. Despite detecting the presence of EBV and CMV by PCR in a portion of patients, few patients received specific therapy, likely because their overall clinical picture was more consistent with an underlying autoimmune or malignant process rather than secondary to EBV or CMV infection. In all MAS patients, the viremia was thought to represent reactivation from immune dysregulation rather than an incident infection. The single MAS patient with CMV viremia was the only patient to receive concomitant antiviral therapy in addition to immunosuppression. Further understanding of the role of these infections in triggering HLH and the implications on treatment is an important area for further research. Although infections can be triggers for other etiologies of HLH, in MAS and mHLH infection is thought to be an epiphenomenon. Regardless, infections should be treated, especially in MAS since concomitant infections in rheumatologic patients on immunosuppressive therapies is not uncommon.

Although we only assessed MAS in adult patients, the disease process is perhaps most typically associated with juvenile idiopathic arthritis (JIA). Unlike adult MAS, MAS associated with JIA has specific classification criteria that require a ferritin $>684 \mathrm{ng} / \mathrm{mL}$ and at least two of the following: thrombocytopenia, elevated AST, elevated triglycerides, or low fibrinogen [40]. Future studies will be needed to assess the validity of these diagnostic criteria in an adult population.

Our study was limited by the small sample size; however, this was not unexpected given the rarity of these diseases. It is conceivable that we may have missed some cases due to coding errors. We suspect many cases of these diseases were misdiagnosed as other diseases that mimic MAS and mHLH as described above. Additionally, we encountered some missing data given the retrospective nature of the design.

\section{Conclusion}

While MAS and mHLH exist on the same spectrum of disease, their distinct triggers and treatment algorithms make differentiating the two processes essential. Our research shows that immunologic laboratory values, most notably platelet count, as well as clinical features, including the absence of hepatomegaly, may help distinguish these two disease states, and as a result, help guide clinical decision making early on in the disease process. Future research into differentiating MAS from other triggers for $\mathrm{HLH}$, including infection, as well as research with larger sample sizes, will be important to further elucidate this important distinction.

Author contributions GSD: substantial contributions to the conception and design of the work; acquisition, analysis and interpretation of data for the work; drafting the work for important intellectual content; final approval of the version to be published. WSD: substantial contributions to the conception and design of the work; acquisition, analysis and interpretation of data for the work; revising it critically for important intellectual content; final approval of the version to be published. KVS: substantial contributions to the conception and design of the work; acquisition, analysis and interpretation of data for the work; revising it critically for important intellectual content; final approval of the version to be published.

Funding None.

\section{Declarations}

Conflict of interest SDW and SDG declare that they have no conflicts of interest. VCK: no direct conflicts of interest. Dr. Kyttaris is a grant recipient of Exagen Diagnostics for unrelated work.

Ethical approval This study was approved by the institutional review board and we certify that the study was performed in accordance with the ethical standards as laid down in the 1964 Declaration of Helsinki and its later amendments. Data for this manuscript are not openly shared. Ethics approval: IRB: 2018P00703. Date: 3/16/2020. Institution: Beth Israel Deaconess Medical Center.

\section{References}

1. Kumakura S, Murakawa Y (2014) Clinical characteristics and treatment outcomes of autoimmune-associated hemophagocytic syndrome in adults. Arthritis Rheumatol 66:2297-2307. https:// doi.org/10.1002/art.38672

2. Daver N, McClain K, Allen CE et al (2017) A consensus review on malignancy-associated hemophagocytic lymphohistiocytosis in adults. Cancer 123:3229-3240. https://doi.org/10.1002/cncr. 30826 
3. Crayne CB, Albeituni S, Nichols KE, Cron RQ (2019) The immunology of macrophage activation syndrome. Front Immunol 10:119. https://doi.org/10.3389/fimmu.2019.00119

4. Schulert GS, Grom AA (2015) Pathogenesis of macrophage activation syndrome and potential for cytokine-directed therapies. Annu Rev Med 66:145-159. https://doi.org/10.1146/annur ev-med-061813-012806

5. Zhang K, Jordan MB, Marsh RA et al (2011) Hypomorphic mutations in PRF1, MUNC13-4, and STXBP2 are associated with adult-onset familial HLH. Blood 118:5794-5798. https:// doi.org/10.1182/blood-2011-07-370148

6. Bracaglia C, Prencipe G, De Benedetti F (2017) Macrophage activation syndrome: different mechanisms leading to a one clinical syndrome. Pediatr Rheumatol Online J 15:5. https:// doi.org/10.1186/s 12969-016-0130-4

7. Brisse E, Matthys P, Wouters CH (2016) Understanding the spectrum of haemophagocytic lymphohistiocytosis: update on diagnostic challenges and therapeutic options. Br J Haematol 174:175-187. https://doi.org/10.1111/bjh.14144

8. Morimoto A, Nakazawa Y, Ishii E (2016) Hemophagocytic lymphohistiocytosis: pathogenesis, diagnosis, and management. Pediatr Int 58:817-825. https://doi.org/10.1111/ped.13064

9. Fardet L, Galicier L, Lambotte O et al (2014) Development and validation of the HScore, a score for the diagnosis of reactive hemophagocytic syndrome. Arthritis Rheumatol 66:2613-2620. https://doi.org/10.1002/art.38690

10. Hayden A, Park S, Giustini D, Lee AY, Chen LY (2016) Hemophagocytic syndromes (HPSs) including hemophagocytic lymphohistiocytosis (HLH) in adults: a systematic scoping review. Blood Rev 30:411-420. https://doi.org/10.1016/j.blre. 2016.05.001

11. Henter JI, Horne A, Arico M et al (2007) HLH-2004: diagnostic and therapeutic guidelines for hemophagocytic lymphohistiocytosis. Pediatr Blood Cancer 48:124-131. https://doi.org/10. 1002/pbc. 21039

12. Davi S, Consolaro A, Guseinova D et al (2011) An international consensus survey of diagnostic criteria for macrophage activation syndrome in systemic juvenile idiopathic arthritis. J Rheumatol 38:764-768. https://doi.org/10.3899/jrheum.100996

13. Eloseily EMA, Minoia F, Crayne CB, Beukelman T, Ravelli A, Cron RQ (2019) Ferritin to erythrocyte sedimentation rate ratio: simple measure to identify macrophage activation syndrome in systemic juvenile idiopathic arthritis. ACR Open Rheumatol 13:345-349. https://doi.org/10.1002/acr2.11048

14. Gavand PE, Serio I, Arnaud L et al (2017) Clinical spectrum and therapeutic management of systemic lupus erythematosusassociated macrophage activation syndrome: a study of 103 episodes in 89 adult patients. Autoimmun Rev 16:743-749. https:// doi.org/10.1016/j.autrev.2017.05.010

15. Liu AC, Yang Y, Li MT et al (2018) Macrophage activation syndrome in systemic lupus erythematosus: a multicenter, casecontrol study in China. Clin Rheumatol 37:93-100. https://doi. org/10.1007/s10067-017-3625-6

16. Hot A, Toh ML, Coppere B et al (2010) Reactive hemophagocytic syndrome in adult-onset Still disease: clinical features and long-term outcome: a case-control study of 8 patients. Medicine (Baltimore) 89:37-46. https://doi.org/10.1097/MD.0b013e3181 caf 100

17. Lerkvaleekul B, Vilaiyuk S (2018) Macrophage activation syndrome: early diagnosis is key. Open Access Rheumatol 10:117128. https://doi.org/10.2147/OARRR.S151013

18. Aringer M, Costenbader K, Daikh D et al (2019) 2019 European league against rheumatism/American College of Rheumatology classification criteria for systemic lupus erythematosus. Arthritis Rheumatol 71:1400-1412. https://doi.org/10.1002/art.40930
19. Yamaguchi M, Ohta A, Tsunematsu T et al (1992) Preliminary criteria for classification of adult Still's disease. J Rheumatol 19:424-430

20. Lundberg IE, Tjarnlund A, Bottai M, The International Myositis Classification Criteria Project Consortium (2017) 2017 European league against rheumatism/American College of Rheumatology classification criteria for adult and juvenile idiopathic inflammatory myopathies and their major subgroups. Ann Rheum Dis 76:1955-1964

21. Gladman DD, Ibañez D, Urowitz MB (2002) Systemic lupus erythematosus disease activity index 2000. J Rheumatol 29:288-291

22. Takahashi N, Chubachi A, Kume M et al (2001) A clinical analysis of 52 adult patients with hemophagocytic syndrome: the prognostic significance of the underlying diseases. Int J Hematol 74:209-213. https://doi.org/10.1007/BF02982007

23. Borgia RE, Gerstein M, Levy DM, Silverman ED, Hiraki LT (2018) Features, treatment, and outcomes of macrophage activation syndrome in childhood-onset systemic lupus erythematosus. Arthritis Rheumatol 70:616-624. https://doi.org/10.1002/art. 40417

24. Diack ND, Kane BS, Fall S et al (2020) Adult hemophagocytic lymphohistiocytosis in sub-saharan area: a retrospective study of 26 cases. Cureus 12:e7258. https://doi.org/10.7759/cureus.7258

25. Hunag Z, Jia Y, Zuo Y, Wu J, Lu A, Zhang L (2020) Malignancyassociated hemophagocytic lymphohistiocytosis in children: a 10 -year experience of a single pediatric hematology center. Hematology 25:389-399. https://doi.org/10.1080/16078454.2020.18335 05

26. Lehmberg K, Pink I, Eulenburg C, Beutel K, Maul-Pavicic A, Janka G (2013) Differentiating macrophage activation syndrome in systemic juvenile idiopathic arthritis from other forms of hemophagocytic lymphohistiocytosis. J Pediatr 162:1245-1251. https://doi.org/10.1016/j.jpeds.2012.11.081

27. Tabata C, Tabata R (2012) Possible prediction of underlying lymphoma by high sIL-2R/ferritin ratio in hemophagocytic syndrome. Ann Hematol 91:63-71. https://doi.org/10.1007/ s00277-011-1239-7

28. Allen CE, Yu X, Kozinetz CA, McClain KL (2008) Highly elevated ferritin levels and the diagnosis of hemophagocytic lymphohistiocytosis. Pediatr Blood Cancer 50:1227-1235. https://doi.org/ $10.1002 /$ pbc. 21423

29. Lin TF, Ferlic-Stark LL, Allen CE, Kozinetz CA, McClain KL (2011) Rate of decline of ferritin in patients with hemophagocytic lymphohistiocytosis as a prognostic variable for mortality. Pediatr Blood Cancer 56:154-155. https://doi.org/10.1002/pbc.22774

30. La Rosée P, Horne A, Hines M et al (2019) Recommendations for the management of hemophagocytic lymphohistiocytosis in adults. Blood 133:2465-2477. https://doi.org/10.1182/blood. 2018894618

31. Gilboa M, Bornstein G, Ben-Zyi I, Grossman C (2020) Macrophage activation syndrome complicating rheumatic diseases in adults: case-based review. Rheumatol Int 40:663-669. https://doi. org/10.1007/s00296-019-04393-7

32. Carter SJ, Tattersall RS, Ramanan AV (2019) Macrophage activation syndrome in adults: recent advances in pathophysiology, diagnosis and treatment. Rheumatology (Oxford) 58:5-17. https:// doi.org/10.1093/rheumatology/key006

33. Monteagudo LA, Boothby A, Gertner E (2020) Continuous intravenous anakinra infusion to calm the cytokine storm in macrophage activation syndrome. ACR Open Rheumatol 2:276-282. https://doi.org/10.1002/acr2.11135

34. Miettenun PM, Narendran A, Jayanthan A, Behnrens EM, Cron RQ (2011) Successful treatment of severe paediatric rheumatic disease-associated macrophage activation syndrome with interleukin-1 inhibition following conventional immunosuppressive 
therapy: case series with 12 patients. Rheumatology (Oxford) 50:417-419. https://doi.org/10.1093/rheumatology/keq218

35. Grom AA, Horne A, De Benedetti F (2016) Macrophage activation syndrome in the era of biologic therapy. Nat Rev Rheumatol 12:259-268. https://doi.org/10.1038/nrrheum.2015.179

36. Marsh RA (2018) Epstein-Barr virus and hemophagocytic lymphohistiocytosis. Front Immunol 8:1902. https://doi.org/10.3389/ fimmu.2017.01902

37. Soy M, Atagündüz P, Atagündüz I, Sucak GT (2021) Hemophagocytic lymphohistiocytosis: a review inspired by the COVID-19 pandemic. Rheumatol Int 41:7-18. https://doi.org/10.1007/ s00296-020-04636-y

38. Kollipara V, Hussain S, Franco-Palacios D, Sofi U (2019) A case series of endemic infections associated hemophagocytic lymphohistiocytosis (HLH) mimicking severe sepsis syndrome. Respir Med Case Rep 27:100854. https://doi.org/10.1016/j.rmcr.2019. 100854
39. Johnson TM, Brown MS, Rabbat M, Slim J (2017) Hemophagocytic lymphohistiocytosis associated with anaplasmosis. J Glob Infect Dis 9:76-78. https://doi.org/10.4103/jgid.jgid_116_16

40. Ravelli A, Minoia F, Daví S, Paediatric Rheumatology International Trials Organisation, the Childhood Arthritis and Rheumatology Research Alliance, the Pediatric Rheumatology Collaborative Study Group, and the Histiocyte Society et al (2016) 2016 Classification criteria for macrophage activation syndrome complicating systemic juvenile idiopathic arthritis. Arthritis Rheumatol 68:566-576. https://doi.org/10.1002/art.39332

Publisher's Note Springer Nature remains neutral with regard to jurisdictional claims in published maps and institutional affiliations. 